\title{
Challenges and Opportunities: Mobile Broadband
}

\author{
Rahul Gupta and Shantipriya Parida,
}

\begin{abstract}
Mobile broadband traffic continues to grow exponentially as subscribers increasingly turn to their mobile devices as their primary access to Internet services and applications. New Smartphones, tablets, and machine to-machine devices are providing a compelling mobile experience by allowing people to engage their social networks, conduct business, and manage their day-to-day activities.

This paper gives an insight into the background, current trend, challenges and opportunities lies in mobile broadband for service providers. The objectives of this paper are: -

To help operators/service providers to understand the challenges due to continuous MBB traffic growth and the steps needed to overcome these challenges. Operators need to plan their network investment accordingly and establish themselves by providing better Quality of Service $(\mathrm{QoS})$ experience \& personalized services to end subscriber.

It also covers latest industry data reports showing the future Mobile Broadband Evolution (MBB), analyzed different dimensions and identifies the potential areas to focus in the MBB era to support the argument.
\end{abstract}

Index Terms-Mobile broadband, long term evolution, smart broadband, cloud service, monetization.

\section{INTRODUCTION}

The mobile broadband rapidly changing the way people access the internet across the globe. It changed the way people live, learn, work and collaborate. Now the internet trend is shifting from desktop to "on-the-go" experience.

As the broadband/internet trend is changing rapidly, the mobile network traffic is shifting from voice to data and the data traffic is already exceeded the voice in December 2009. In countries like India, South Africa, Nigeria the mobile broadband penetration contribute to the Gross Domestic Product (GDP) of the country. The GDP contribution is not only limited to growing developing country, it also impact to countries like Taiwan, china and United States of America (USA). Fig. 1 illustrates the growth trend of mobile broadband with respect to fixed broadband [1].

\section{BACKGROUND}

This section describes the evolution path of the mobile technologies from first generation $(1 \mathrm{G})$ to fourth generation (4G). Fig. 2 below is showing the evolution trend:

\section{A. $1 G$}

In the 1970s foundation was laid with a design that has known as $1 \mathrm{G}$. 1G was implemented based on analog

Manuscript received January 11, 2013; revised April 1, 2013.

The authors are with the Huawei Technologies India Pvt Ltd, The Leela Palace, Old Airport Road, Bangalore 560012 INDIA (e-mail: shantipriya.parida@gmail.com,g007.rahul@gmail.com). technology and the basic cellular structure of mobile communication.

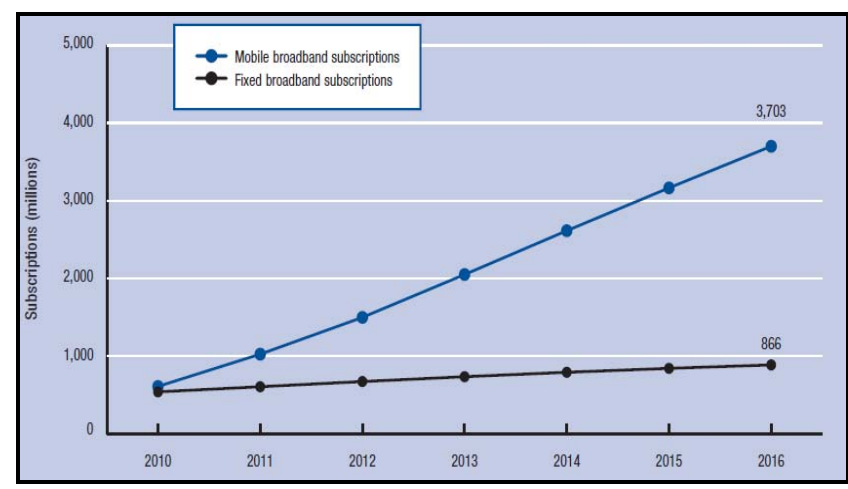

Fig. 1. Forcast: global broadband subscriptions [1]

\section{B. $2 G$}

The second generation (2G) systems designed in the 1980s particularly used for voice applications at a low speed for digital circuit switched networks.

\begin{tabular}{|c|c|c|c|c|c|}
\hline Technolody & 16 & 26 & 2.50 & 38 & 40 \\
\hline Design Began & 1970 & 1980 & 1985 & 1990 & 2000 \\
\hline Implementation & 1984 & 1991 & 1999 & 2002 & $2010 ?$ \\
\hline Service & $\begin{array}{l}\text { Analog voice, } \\
\text { synchronous data } \\
\text { to } 9.6 \text { kbps }\end{array}$ & $\begin{array}{l}\text { Digital wice, } \\
\text { short } \\
\text { messages }\end{array}$ & $\begin{array}{l}\text { Higher capacity, } \\
\text { pachetized data }\end{array}$ & $\begin{array}{l}\text { Higher capacity, } \\
\text { broadband data } \\
\text { up to } 2 \text { Mbps }\end{array}$ & $\begin{array}{l}\text { Higher capacity. } \\
\text { completely IP. } \\
\text { oriented, multimedia. } \\
\text { data to hundreds } \\
\text { of megabits }\end{array}$ \\
\hline Stanciards & $\begin{array}{l}\text { AMPS, TACS, } \\
\text { NMT, etc. }\end{array}$ & $\begin{array}{l}\text { TDMA, CDMA, } \\
\text { GSM, PDC }\end{array}$ & $\begin{array}{l}\text { GPRS, EDGE, } \\
\text { 1XRTT }\end{array}$ & $\begin{array}{l}\text { WCDMA, } \\
\text { CDMA2000 }\end{array}$ & Single standard \\
\hline Data Bandwidth & $1.9 \mathrm{kbps}$ & $14.4 \mathrm{kbps}$ & $384 \mathrm{kbps}$ & 2 Mbps & 200 Mbps \\
\hline Mutiplexing & FDMA & TDMA, CDMA & TDMA, CDMA & CDMA & CDMA? \\
\hline Core Network & PSTN & PSTN & $\begin{array}{l}\text { PSTN, } \\
\text { packet network }\end{array}$ & Packet network & Internet \\
\hline
\end{tabular}

Fig. 2. Technology evolution trend [2]

\section{C. $2.5 \mathrm{G}$}

$2.5 \mathrm{G}$ is a cellular wireless technology developed in between its predecessor, $2 \mathrm{G}$, and its successor, $3 \mathrm{G}$. A 2.5G system may make use of $2 \mathrm{G}$ system infrastructure, but it implements a packet-switched network domain in addition to a circuit-switched domain. General Packet Radio Service (GPRS) provides data rates of 56-114 kbit/second.

\section{D. $3 G$}

3G system was introduced during 1990s to eliminate previous incompatibilities and become a truly global system. The $3 \mathrm{G}$ system supports higher quality voice channels, as well as broadband data capabilities, up to 2 Mbps.

\section{E. $4 G$}

Long Term Evolution (4G/LTE) Technology is introduced to meet the increased demand for higher access speed multimedia communication nowadays. The 4G/LTE networks can download data at speeds between 5 and 12 megabits enough for smooth streaming for live video and better network for mobile devices like Smartphones and 
Tablets [2].

\section{Challenges In Mobile BRoAdBAnd}

\section{A. End to End Quality of Service (E2E QoS) Delivery}

As the users are increasingly aware of the bandwidth, data rate, coverage and the mobile broadband service, operators must deliver consistent, high-quality, and seamless mobile broadband experience that meets or exceeds their expectation. The Fig. 3 shows the combined approach for delivering consistent user experience which is required to improve the overall data performance at cell site \& at cell edges particularly indoors.

However, in certain scenarios the demand for mobile broadband is now increasingly challenging such as:

- Large outdoor hotspots, such as town squares and commercial streets with high traffic demand

- Large, isolated indoor hotspots, such as businesses, hotels and shopping malls

- Localized, indoor hotspots such as small offices, restaurants and retail outlet

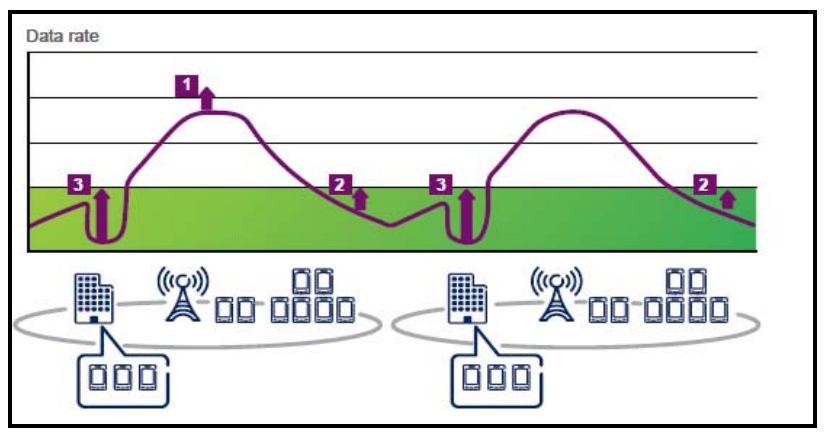

Fig. 3. Combine approach to delivering consistent user experience. (1) better overall cell-site performance (2) enhance cell edge data rates (3) increase indoor data rates [3]

The above challenges can be overcome by designing solutions where the small cells complement macro cells which will help operators to meet the growing demand for mobile broadband. However, to make this happen, a number of challenges need to be overcome.

1) Backhaul challenge: The Backhaul has gain importance because of the following factors:

- Small cells compliments densified macro cells

- Increase in the number of radio nodes

- In Smartphones era, the unexpected traffic produced network congestion and outages that created major customer dissatisfaction. As much as $50 \%$ of the problems were attributable to inadequate backhaul.

Networks with small cell sites require backhaul solutions that can use a selection of physical transmission media, including microwave, fiber, copper lines and wireless connectivity. Operators need to rethink their backhaul investments as they deploy small cells and LTE capacity and to make balance between utilizing the existing infrastructure and investing in dedicated backhaul transmission that results in higher overall network performance. This decision may affect installation costs and the time needed for site acquisition and installation.
2) Site setup challenge: The operators need to implement flexible base-station site solution to obtain maximum value from the radio spectrum. The operators need to consider new partners such as municipalities, retailers and external agencies as alternatives for site location instead of deals made with landlords and tower-approval committees.

3) Scalability challenge: Deployments that involve a significant number of small radio base stations change the economics of mobile network rollouts. The operator need holistic planning and phased migration to scale up in a given network area.

The complexity of a network from radio perspective composed of multiple layers and radio technologies could easily become unmanageable unless designed carefully. The effective interworking between small and macro cells needs seamless move from one radio-access technology to another maintaining maximum coverage with proper resource utilization [3].

\section{B. Drop in $M B B$ Revenue}

Currently, one of the important business challenges that carriers are facing is "revenue growth not keeping pace with the growth in data volumes". The gap between mobile traffic growth and revenue growth is expanding as shown in Fig. 4. Service provider's needs to apply innovative ways to monetize mobile broadband, attract and retain customers, and efficiently manage network resources [4].

Service providers can leverage on the assets like mobile broadband traffic growth, Smartphones \& their applications, Over the Top (OTT) players, Consumer behavior etc. Service providers can deliver innovative, revenue generating services by combining these assets with sophisticated monetization tools such as policy-based QoS and quota features, identity management, open application interfaces, and performance analytics [5].

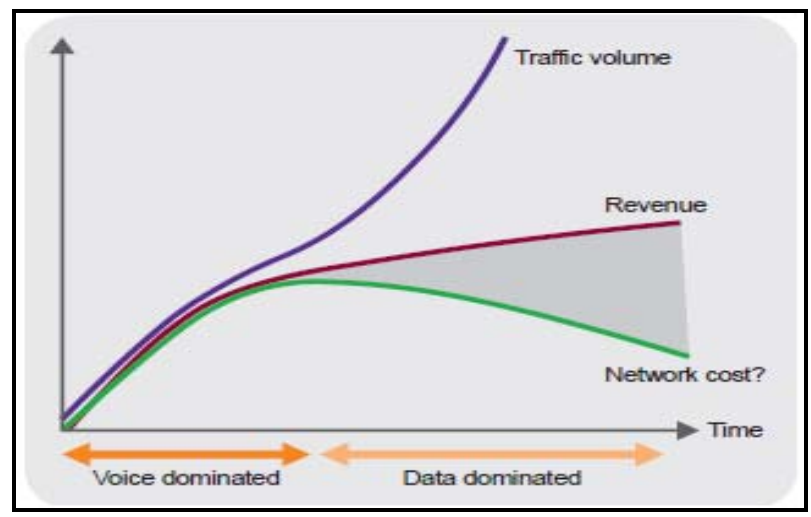

Fig. 4. Traffic V/S revenue growth [4]

\section{MBB CURRENT TREND}

In year 2011, mobile data traffic growth rate was higher than anticipated. Last year's growth rate projection was $131 \%$ \& estimation is that global mobile data traffic grew 133 percent in 2011. It is also estimated that Mobile data traffic will grow at a compound annual growth rate (CAGR) of 78 percent $\&$ will surpass 10 exabytes/month in 2016 . The Fig. 5 shows the expected growth trend per month by 2016 [6]. 


\section{A. Increase Bandwidth Consumption}

It has been seen in past some years that the bandwidth utilization has increased steeply due to emerging user behaviors brought by the increased popularity of data hungry mobile devices [e.g. Smartphones, Personal Digital Assistance (PDA)] [7].

Another aspect of this tremendous traffic growth mentioned above is the influx of mobile applications in our lives. Some of the applications like gaming, Voice over IP (VOIP), file sharing, Instant Messaging (IM), Video are amongst favorites and easily available in the Android or iPhone Operating System (iOS) market. Mobile video is expected to increase 25 -fold by 2016, and will account for over 70 percent of total mobile data traffic. The distribution of expected data usage between different applications till 2016 is shown in Fig. 7 [6].

1) Device innovation: Device innovation from cellular phones to smart phones is one of the primary reasons for the broadband traffic growth. In 2011, the typical Smartphone generated 35 times more mobile data traffic than the typical basic-feature cell phone [6]. Smartphones will generate averagely $2.6 \mathrm{~GB}$ of traffic per month in 2016, which is 17-fold increase over the 2011 average of $150 \mathrm{MB}$ per month. The Fig. 6 shows the growth trend of the different device types [6].

As these devices continue to demand more data, it is evident that current $3 \mathrm{G}$ and $4 \mathrm{G}$ networks with better QoS alone cannot handle the upswing in traffic [8].

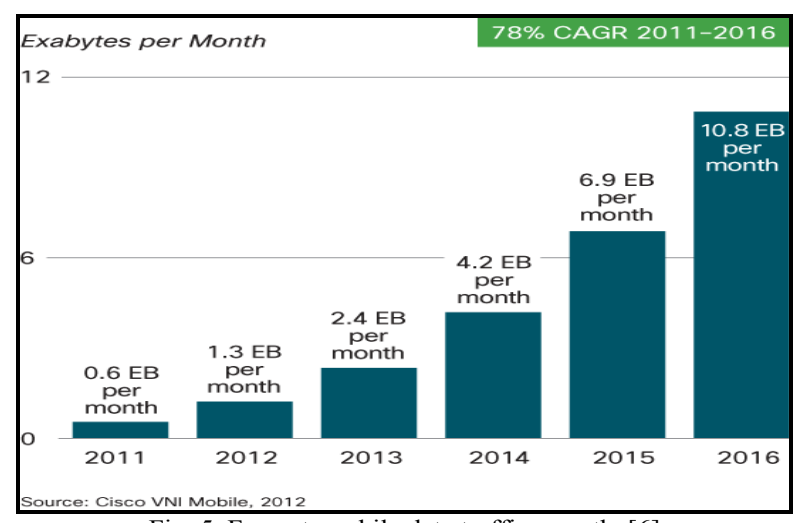

Fig. 5. Forcast: mobile data traffic growth. [6]

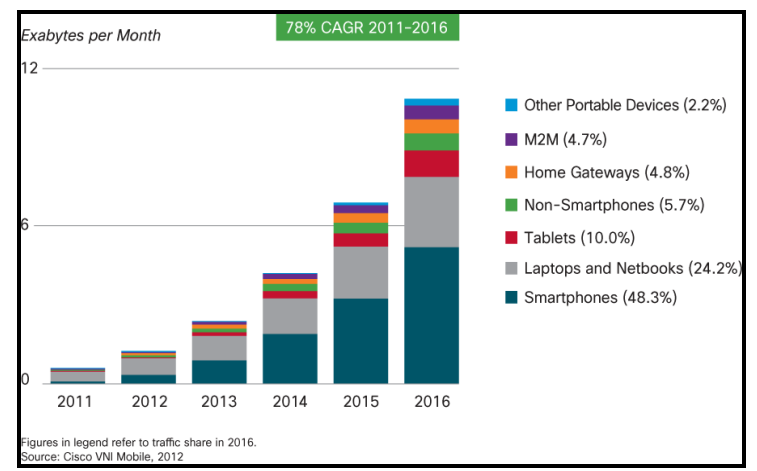

Fig. 6. Forcast: data traffic growth rate by devices [6]

2) Popularity of smart phone applications: With the introduction of Smartphones and tablets, the applications running on these devices have become very popular amongst subscribers like games, IM, OTT applications etc. Mobile operating systems like Apple's iOS and Google's Android platforms fueling an explosion of diversified apps built on their foundations. The open source culture adopted by these mobile software vendors has penetrated the Smartphone market and allows developer to showcasing their wares. With such easy tools and platforms provided for the developers many applications have been developed over a period of time and added to these application markets. Apple recently announced the availability of over 700,000 such apps. Fig. 7 is showing the expected mobile applications growth trend [8].

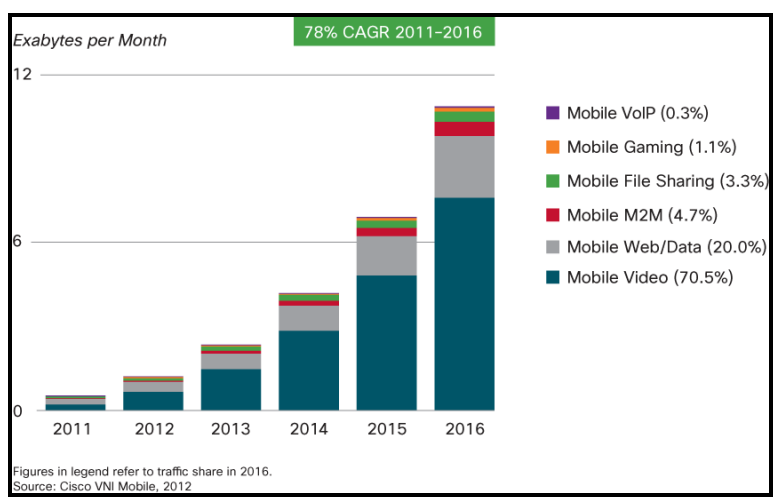

Fig. 7. Forcast: mobile applications traffic growth rate [6]

\section{B. Long Term Evolution Technology}

The evolving LTE technology will increase the existing network performance beyond anything and all major industry players are now focusing on this area. LTE technology offers a number of distinct advantages such as increased performance attribute, high peak data rates, low latency, and greater efficiencies over other wireless technologies. Improved performance and increased spectral efficiency will allow wireless carriers to offer higher quality products and services to their customers [9]. As per Analysis Research forecast, the number of LTE subscribers could exceed 400 million by 2015 . The Fig. 8 depicts the subscriber's growth expected by 2015 [10].

Carriers are looking for new revenue streams, because revenues are stagnating, particularly in voice services. LTE offers new tariff options \& strengthens user-paid revenues with new IP services, such as multi-screen video and location-based offerings and generate additional revenues from nontraditional services, such as targeted advertising, content brokering and e-commerce transactions. As per Analysis Research forecast, LTE revenue could exceed EUR 150 billion globally by 2015 . Fig. 9 depicts the LTE data revenue growth by service [10].

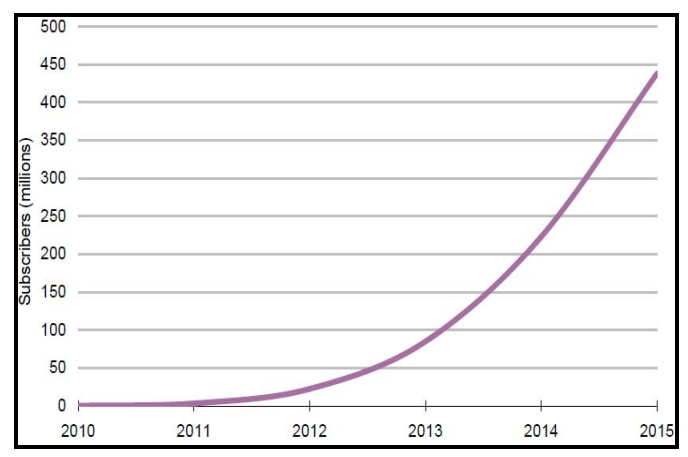

Fig. 8. Forecast: LTE subscribers growth [10]

\section{SCOPE AND OPPORTUNITIES}

Mobile network traffic had been shifted from voice to data during last decade, and in December 2009, for the first time data traffic exceeded the volume of voice calls across the 
world's mobile networks [1].

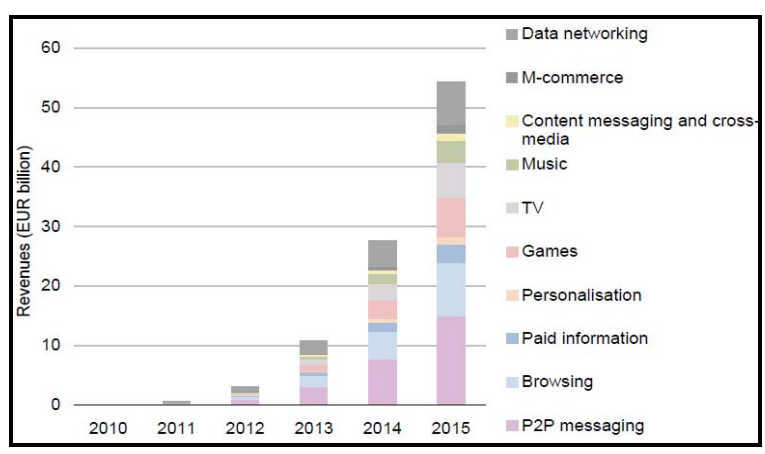

Fig. 9. Forecast: global data LTE revenues by service [10]

As mobile broadband networks are increasingly able to deliver rich Internet access and data services, mobile data traffic will continue its phenomenal growth. Fig. 10 shows the MBB expected growth in coming years [1] [11]. The mobile broadband can offer the followings to operator to leverage with comparison to traditional broadband solution.

\section{A. Transformation from Mobile to Smart Broadband}

Based on the substantial shift from fixed broadband to mobile broadband among certain niche user segments, it is observed that most users want to experience the best broadband connection speed/quality for their money.

The majority of users will benefit from a simple, hybrid broadband solution that allows them to connect based on location, connectivity options available, devices and applications, budget/cost preferences etc.

From an operator's perspective, it could provide a number of positive benefits like:

- Attract more customers with easy usage, ubiquitous coverage, and cost effectiveness.

- Allows operators to charge more for bundled services $\&$ could generate higher broadband monthly Average revenue per user (ARPU).

- Good customer experience to yield positive benefits on customer retention [12].

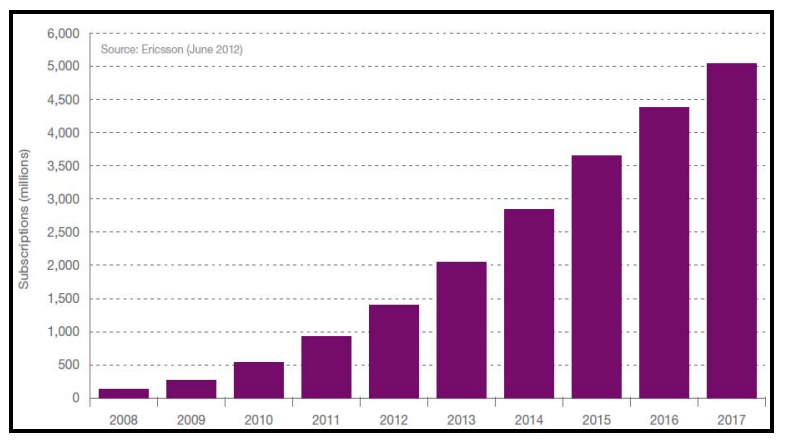

Fig. 10. Forcast: Mobile broadband subscriptions Trends [11]

\section{B. Monetization of Data Services}

The continuous increase in bandwidth consumption, coupled with every device being connected, eventually leads to a world where all networks will converge over IP and will become an all IP network with no IP scarcity - and that shift will dramatically impact how services are ordered, delivered, and billed across all providers, including broadband [7]. According to Ovum Research, there will be three billion big-and small-screen mobile broadband connections globally by 2015 [5].

Below mentioned are some use cases that illustrate how service providers can implement an intelligent approach to monetize mobile broadband, including: personalized service tiers, seamless service delivery, adding value to OTT applications:

1) Seamless delivery and service (one customer - many devices) In today's market, consumer expects a seamless experience whether they use a Personal Computer (PC), Television (TV), or stream content through their Xbox, Smartphone or iPAD [7]. This allows operators to offer customer-centric mobile broadband services by providing customers with one plan that spans many devices, for example - shared data plan, Family Plan etc. This approach combines subscriber and device data management to assign multiple device identities to one customer profile [5].

This simplified plans approach will reduce subscriptions, accelerate the monetization of mobile broadband services, provide greater transparency into group usage and also reduce costs by simplifying and reducing billing requirements [5].

2) Moving towards personalized packages: The days have gone where the consumers have to choose from what Content Provider (CP)/Service Provider (SP) offers to them. Now consumers are expecting plans tailored to their specific usage and preferences and pay accordingly. As a result, the operator has to offer a new breed of personalized services that the subscriber is willing and happy to pay for. The personalized services virtually offer endless service possibilities for the operators [13]. Operator can combine consumer demands \& network intelligence (policy management) to meet the requirements of different customer and market segments with personalized service tiers like Bandwidth tiers, Application based tiers, Speed based tiers, time based tiers, device based tiers etc [5]. The evolution trend from usage based plans to value based plans is shown in Fig. 11.

3) Monetize OTT services usage Many mobile operators are facing challenge to handle OTT services as these services are attracting subscribers away from using the operator services because of their free nature. The challenges offered by these OTT applications for mobile operators are:

- How to handle the increased data traffic

- How to monetize that increased burden

For example, the OTT messaging players such as iMessage, WhatsApp, is pushing mobile operators' messaging profits down in many developed markets as consumers opt to use these OTT services for delivering their messages \& save their money.

In this realm, it is clearly visible that mobile operators have to change their data charging strategies from "usage-based" to "value-based" pricing service plans by applying application based charging, create tiered service plans to overcome these challenges.

Application based charging allows subscribers to choose a plan that most appropriately suits his/her needs and offers many benefits to operators such as Increase ARPU, Increase customer loyalty, differentiate service offering etc[14].

4) Network analytics: Network Analytics help operators understand the network usage and data consumption trend (per subscriber basis) in their network. Operators should use 
detailed network analytics function to understand the behaviors of the data users. Based on the reports, operators can optimize the network performance and also can offer personalized packages based on per subscriber usage trend.

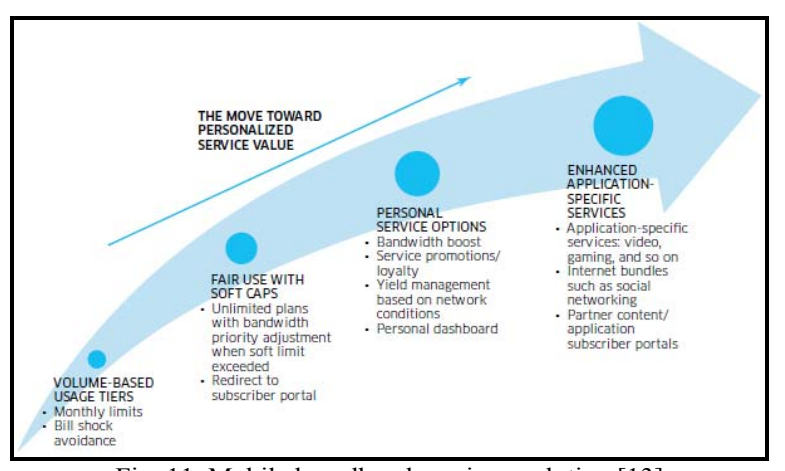

Fig. 11. Mobile broadband service evolution [13]

\section{Cloud-Based Services}

The rapid increase in the number of connected consumer electronics and industry-specific machine-to-machine (M2M) devices will initiate the need for industry-specific cloud applications and non-traffic-based charging models [15].

Cloud services offer multiple opportunities for operators to empower their customers. It depends on a combination of both Information Technology (IT) and telecoms expertise. For operating cloud services effectively, operator needs to emphasize on network reach, control and complex service-management capabilities [16].

Cloud services will be characterized by everything being connected. Revenue will increase by meeting the needs of the foresee more than 50 billion connected devices [15].

As we know mobile devices have memory and speed limitations, cloud services will allow devices to store \& access content and applications in data centers in the cloud, instead of locally [15]. Cloud applications and services such as Netflix, YouTube, Pandora, and Spotify allow mobile users to overcome the limitations of mobile devices. A Smartphone user adopting Netflix, Pandora etc will generate more than twice the traffic generated by a Smartphone user adopting only email and web applications as shown in Fig. 12 [6].

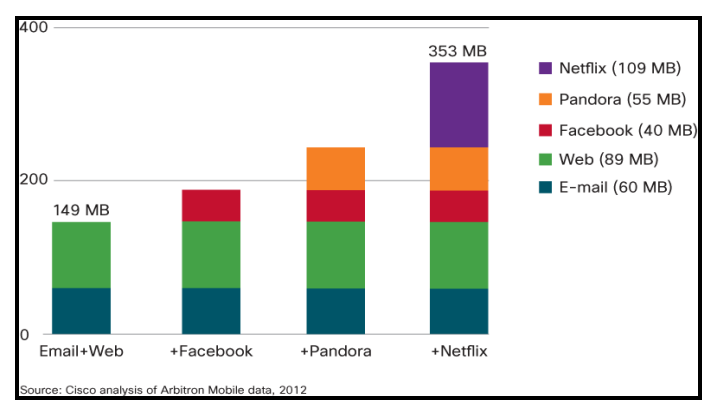

Fig. 12. Cloud media applications multiply smartphone traffic [6]

\section{CONCLUSION}

Mobile broadband opening up new internet experiences, unlocking new opportunities and it is becoming a force to change the socioeconomic levels in every corner of the globe [1].

The current research conveys that subscribers want operators to be more trustworthy, uncomplicated and honest.
We firmly believe that operators could enhance their growth chances by taking the approaches proposed in this paper [17]. The benefits for operators include:

- Increased goodwill

- Increased loyalty and reduced churn

- Increased usage and revenue [17]

Mobile broadband uniquely provides this potential to empower individuals across the world as never before. In many profound ways, this technology is an economic development tool for the 21 st century. Apart from connecting people to each other, mobile broadband also paves a way for highly personalized internet and computing experience [1].

\section{REFERENCES}

[1] W. Bold and W. Davidson, "Mobile broadband: redefining internet access and empowering individuals," The Global Information Technology Report 2012, World Economic Forum, pp. 67-77, 2012.

[2] 4G-Quadruple Play High Speed Mobile Broadband Technologies, White Paper, Tech Mahindra, 2008.

[3] Heterogeneous Networks, White Paper, Ericsson, Feb 2012.

[4] Enabling mobile broadband growth - Evolved Packet Core, White Paper, Nokia Siemens Networks, 2009.

[5] An Intelligent Approach to Monetizing Mobile Broadband, White Paper, Tekelec, 2012.

[6] Cisco Visual Networking Index: Global Mobile Data Traffic Forecast Update, 2011-2016, White Paper, Cisco, Feb 2012.

[7] Real-Time Services for the Broadband Industry, White Paper, CSG International, Jul 2011.

[8] Mobile Broadband Explosion, White Paper, Rysavy Research, Sep 2011.

[9] The Future of Mobile Broadband Technology, White Paper, Verizon Wireless, 2009.

[10] Global Mobile Broadband: Market potential for 3G LTE, Analysys Research Limited, UMTS Forum, Jan 2008.

[11] Traffic \& Market Report, Ericsson, Jun 2012.

[12] The Fixed/Mobile Broadband Battle: Is it Time for 'Smart Broadband, Cisco Internet Business Solutions Group (IBSG), Nov 2009.

[13] The Delivery of Personal Service Value, Application Note, Alcatel Lucent , 2011.

[14] Optimizing, Monetizing \& Personalizing Operator Networks, Use Cases, Allot, 2011.

[15] Differentiated Mobile Broadband, White Paper, Ericsson, Jan 2011.

[16] The Connected possibilities of Mobile Broadband, White Paper, Huawei, Feb 2012.

[17] Smarter Mobile Broadband, A Consumer Insight Summary Report, Ericsson, Jun 2012.

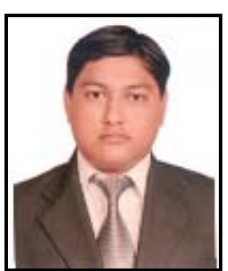

Rahul Gupta is currently working as a senior product engineer in Huawei Technologies India Pvt Ltd. He has completed his Bachelor of Technology (B.Tech) in Electronics \& Communication Engineering (ECE) from Uttar Pradesh Technical University, Uttar Pradesh and also a Cisco Certified Network Professional (CCNA). His area of interest includes Mobile Broadband, Internet Technologies, and Data.

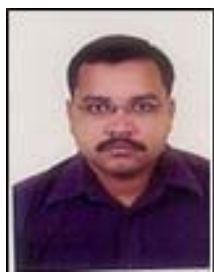

Shantipriya Parida is currently working as a senior solution analyst in Huawei Technologies India Pvt Ltd. He has completed his Master of Computer Application (MCA) and Master of Technology in Computer Science (M.Tech. (CS)) both from Utkal University, Orissa. His area of interest includes Mobile Broadband, Internet Technologies, Machine Learning and Soft Computing. He is a member of IEEE and IACSIT. He also has 6 publications in international conferences and journals. 